\title{
Um Estudo Comparativo entre Simuladores Computacionais para Apoio à Disciplina de Arquitetura e Organização de Computadores
}

\author{
Guilherme Esmeraldo1, Cícero Samuel Mendes1, Lucas Fontes1, Edson Lisboa2
}

1Instituto Federal do Ceará (IFCE) campus Crato - Crato - CE - Brasil 2Instituto Federal de Sergipe (IFS) campus Aracaju - Aracaju - SE - Brasil

guilhermealvaro@ifce.edu.br, mr.samuelmendes@gmail.com, lfonteesc@gmail.com, edson.lisboadacademico.ifs.edu.br

\begin{abstract}
This paper presents a comparative study of simulators used to support the discipline of computer architecture and organization, in order to identify, according to a set of predetermined metrics, their potentialities and deficiencies in the teaching-learning process, as well as they can make possible the acquisition of practical skills, which are fundamental for the professional exercise. According to the results, it was possible to verify that the most of the simulators does not meet the proposed metrics and that there is a need for more efforts to deal accessibility and methodological aspects.
\end{abstract}

Resumo. Este artigo apresenta um estudo comparativo de simuladores utilizados para apoio à disciplina de arquitetura e organização de computadores, a fim de identificar, de acordo com um conjunto de métricas preestabelecidas, suas potencialidades e deficiências no processo de ensinoaprendizagem, bem como se possibilitam a aquisição de habilidades práticas, as quais são fundamentais para o exercício da profissão. De acordo com os resultados, pôde-se constatar que a maioria dos simuladores não atendem às métricas utilizadas e que há necessidade de maiores esforços com o objetivo de tratar aspectos de acessibilidade e metodológicos.

\section{Introdução}

Arquitetura e Organização de Computadores (AOC) é uma disciplina presente em cursos técnicos, tecnológicos e de engenharias, tanto na área de computação, quanto em elétrica/eletrônica. Em geral, seu conteúdo apresenta uma complexidade crescente em função do nível de detalhamento e abrangência a serem consideradas na abordagem pedagógica adotada. Além disso, por se tratar de uma disciplina fundamental em tais áreas, deve estar alinhada às novas tendências tecnológicas, como Computação Paralela e Internet das Coisas [Ghafarian 2016].

Em AOC, uma abordagem metodológica adequada mescla os aspectos teóricos com um conjunto de experimentos que visam, além de sedimentar o conteúdo, o desenvolvimento de habilidades práticas imprescindíveis para os desafios demandados no exercício profissional [Black 2016]. Os aspectos práticos da disciplina podem ser desenvolvidos utilizando laboratórios especializados, os quais pressupõem a disponibilidade de hardware real (componentes eletrônicos, ferramentas, placas de circuitos, modernos equipamentos de testes e medições, etc.), o que podem encarecer o 
ambiente e necessitar de apoio técnico especializado para a sua montagem e manutenção. Para contornar este problema, como uma alternativa aos laboratórios físicos, na literatura tem-se optado pelo uso de simuladores computacionais como ferramenta de apoio ao aprendizado em AOC. Os simuladores são ambientes virtuais de aprendizagem que podem emular computadores completos e viabilizam o estudo de seus componentes, suas funções e interconexões. Ainda na literatura, estão disponíveis diferentes simuladores computacionais, sendo que cada um deles possui suas próprias particularidades [Kurniawan and Ichsan 2017]. Para apoiar o processo de ensinoaprendizagem, a metodologia ideal deve empregar o uso de bons simuladores que apresentem cenários próximos aos reais, mas que possibilitem a interação com $o$ hardware real para o desenvolvimento de habilidades práticas plenas.

Nesse contexto, este trabalho apresenta um estudo comparativo entre simuladores computacionais presentes no estado da arte, com o objetivo de explorar suas potencialidades e deficiências para apoio aos processos de ensino-aprendizagem em Arquitetura e Organização de Computadores.

\section{Materiais e Métodos}

A Sociedade Brasileira de Computação (SBC) definiu um conjunto de diretrizes curriculares para cursos na área de Computação, as quais sugerem os principais tópicos para a disciplina de Arquitetura e Organização de Computadores [Rocha et al. 2005]. De uma forma geral, esses tópicos estão divididos em três grupos: 1) a estrutura dos componentes dos computadores, suas funções e modelos de interconexões; 2) a programação do computador em linguagem de máquina (baixo nível), considerando o conjunto de instruções do computador, a localização dos dados em memória e os diferentes modelos de operações de entrada/saída de dados; e 3) diferentes aspectos concernentes ao desempenho computacional. Neste trabalho, realizou-se inicialmente, com base nesses três grupos de tópicos, uma pesquisa bibliográfica sobre a utilização de simuladores computacionais para apoio à disciplina de Organização e Arquitetura de Computadores.

Visando refinar os resultados da pesquisa, optou-se por selecionar simuladores de sistemas completos [Penna and Freitas 2013], que são aqueles que incluem todos os componentes do computador, como processador, memórias, barramentos e periféricos de entrada/saída. Ressalta-se que, além dos simuladores de sistema completo, encontram-se, na literatura, ferramentas com propósitos específicos: simulação de componentes específicos, como os de memória cache [Cordeiro et al., 2003]; simulação de circuitos digitais em geral, como o apresentado em [Donzellini and Ponta 2007]; e desenvolvimento de projetos profissionais de hardware, como o exposto em [Kleitz 2006]. Ao final desta etapa, selecionou-se um total de 16 simuladores, os quais foram divididos em quatro categorias, de acordo com seu objetivo:

- 10 deles foram desenvolvidos exclusivamente para apoio educacional (BIPIDE [Vieira, Raabe and Zeferino, 2009], COMPSIM [Esmeraldo and Lisboa, 2017], CPU SIM [Skrien, 2001], DIMIPSS [Felix, Souza and Carvalho, 2006], MARIE [Null and Lobur, 2003], MARS [Vollmar and Sanderson, 2006], SIMAEAC [Verona, Martini and Gonçalves, 2009], SIMUS [Silva and Borges, 2017], SOIS [Cruz et al., 2008] e YASS [Mustafa, 2013]);

- 4 deles suportam a execução de sistemas operacionais completos (BOCHS [Bochs, 2017], GEM5 [Butko et al., 2012], MARSS [Patel et al., 2011] e SIMICS [Magnusson et al., 2002] );

- 1 deles é descrito em linguagem de descrição de hardware (HDL) para execução direta em plataformas de hardware reconfigurável (cMIPS [Hexsel and Carmo, 
2013]); e

- O último foi criado para suportar a execução de aplicações de computação intensiva, as quais necessitam de alto desempenho e precisão dos resultados (ESESC [Ardestani and Renau, 2013]).

A próxima etapa consistiu em definir um conjunto de métricas para análise e comparação dos simuladores. Observa-se que, na literatura, há trabalhos que comparam os simuladores computacionais sob diferentes características, como os apresentados em [Penna and Freitas, 2013] [Nikolic et al., 2009] [Akram and Sawalha, 2016] [Wolffe et al., 2002] [Vieira, Raabe and Zeferino, 2009]. Nesses trabalhos, as métricas apontadas, em sua grande maioria, tratam de dois tipos de aspectos, relacionados: 1) aos simuladores em si, como desempenho e precisão de simulação, uso de abstrações na codificação de software dos componentes simulados, interfaces gráficas e licenças de distribuição de software; e 2) ao suporte a recursos mais avançados de simulação, como simulação de diferentes arquiteturas de hardware, projeto de circuitos digitais e recursos de computação paralela. Assim, neste artigo, definiu-se um conjunto de métricas para comparação dos simuladores, com base nos estudos comparativos da literatura, nos tópicos apontados pela SBC e no suporte educacional em AOC (a seção a seguir descreve cada uma das métricas propostas).

Ao final, realizou-se uma análise de cada um dos simuladores selecionados, sob face das métricas de comparação elencadas na etapa anterior. O objetivo consistiu em destacar aqueles com maior potencial de apoio ao processo de ensino-aprendizagem em AOC, bem como identificar quais métricas são fundamentais e aquelas que necessitam de maiores esforços para caracterização de um simulador mais adequado.

\section{Métricas de Comparação}

As métricas de comparação adotadas neste trabalho abrangem:

- Macrogrupos de conteúdos que devem ser trabalhados na disciplina de AOC, segundo a SBC, que são o estudo dos componentes do computador, programação em nível de máquina e análise de desempenho (ressalta-se que cada um desses grupos de tópicos permitiu criar um grupo individual de métricas);

- Aspectos de simulação, visto que, como o simulador tem sido adotado como uma alternativa aos laboratórios especializados, compreende-se que o mesmo torna-se parte fundamental no processo de ensino-aprendizagem;

- Suporte ao desenvolvimento das habilidades práticas, as quais são fundamentais para o exercício da profissão;

- Aspectos de acessibilidade e usabilidade, importantes para permitir o uso do simulador por diferentes tipos de públicos e com diferentes níveis de habilidades;

- Suporte ao processo de ensino-aprendizagem, no qual caracteriza-se o modelo pedagógico proposto pelo uso do simulador.

Essas métricas foram divididas em 7 grupos, cujas descrições são dadas a seguir:

Grupo 1. Componentes do Computador

\begin{tabular}{|c|l|l|}
\hline Id. & \multicolumn{1}{|c|}{ Métrica } & \multicolumn{1}{c|}{ Descrição } \\
\hline G1.1 & Plataforma computacional & $\begin{array}{l}\text { Inclusão dos principais componentes do computador (processador, } \\
\text { memórias principal e cache, e barramento). }\end{array}$ \\
\hline G1.2 & Granularidade & Inclusão de detalhes das características dos componentes. \\
\hline
\end{tabular}


Grupo 2. Programação em Baixo Nível

\begin{tabular}{|c|l|l|}
\hline Id. & \multicolumn{1}{|c|}{ Métrica } & \multicolumn{1}{c|}{ Descrição } \\
\hline G2.4 & Programação em Assembly & Suporte à programação em baixo nível do computador. \\
\hline G2.5 & $\begin{array}{l}\text { Identificação de erros no } \\
\text { programa }\end{array}$ & Apresentação de erros, se presentes no programa em baixo nível. \\
\hline G2.6 & $\begin{array}{l}\text { Ferramentas de suporte à } \\
\text { codificação em baixo nível }\end{array}$ & $\begin{array}{l}\text { Inclusão de mecanismos que simplifiquem a codificação de novos } \\
\text { programas em baixo nível. }\end{array}$ \\
\hline
\end{tabular}

\section{Grupo 3. Simulação}

\begin{tabular}{|c|c|c|}
\hline Id. & Métrica & Descrição \\
\hline G3.7 & Modos de simulação & $\begin{array}{l}\text { Inclusão de diferentes modos de simulação, para que o estudante } \\
\text { possa optar entre simulação detalhada ou com maior desempenho. }\end{array}$ \\
\hline G3.8 & Visualização do programa & $\begin{array}{l}\text { Mecanismos para visualização do estado do programa durante uma } \\
\text { simulação. }\end{array}$ \\
\hline G3.9 & Visualização de componentes & $\begin{array}{l}\text { Visualização dos estados dos componentes (processador, memórias } \\
\text { e periféricos). }\end{array}$ \\
\hline G3.10 & Visualização de comunicação & Visualização da troca de dados entre os componentes. \\
\hline G3.11 & Animação do caminho de dados & $\begin{array}{l}\text { Uso de animações gráficas do caminho de dados do computador, } \\
\text { visando simplificar a compreensão do funcionamento interno do } \\
\text { computador. }\end{array}$ \\
\hline
\end{tabular}

Grupo 4. Análise de Desempenho

\begin{tabular}{|c|l|l|}
\hline Id. & \multicolumn{1}{|c|}{ Métrica } & \multicolumn{1}{c|}{ Descrição } \\
\hline G4.12 & Perfis de simulação & $\begin{array}{l}\text { Mecanismo para registro da sequência de eventos gerados pelos } \\
\text { componentes durante uma simulação, para verificação de gargalos } \\
\text { de desempenho. }\end{array}$ \\
\hline G4.13 & Estatísticas de simulação & $\begin{array}{l}\text { Mecanismo para sumarização dos eventos gerados pelos } \\
\text { componentes durante uma simulação, para verificação em alto nível } \\
\text { de gargalos de desempenho. }\end{array}$ \\
\hline
\end{tabular}

Grupo 5. Apoio ao Desenvolvimento de Habilidades Práticas

\begin{tabular}{|c|c|l|}
\hline Id. & \multicolumn{1}{|c|}{ Métrica } & \multicolumn{1}{c|}{ Descrição } \\
\hline G5.14 & Integração com hardware físico & $\begin{array}{l}\text { Integração do computador virtual com hardware físico, visando } \\
\text { estabelecer cenários semelhantes aos reais em projetos de sistemas } \\
\text { computacionais. }\end{array}$ \\
\hline
\end{tabular}

\section{Grupo 6. Acessibilidade e Usabilidade}

\begin{tabular}{|c|l|l|}
\hline Id. & \multicolumn{1}{|c|}{ Métrica } & \multicolumn{1}{c|}{ Descrição } \\
\hline G6.15 & Interface gráfica & $\begin{array}{l}\text { Inclusão de interface com componentes gráficos para simplificação } \\
\text { da interação com o simulador. }\end{array}$ \\
\hline G6.16 & Personalização da interface & $\begin{array}{l}\text { Inclusão de suporte de ajustes da interface gráfica (personalização } \\
\text { de cores e tamanho da fonte de texto do ambiente de simulação). }\end{array}$ \\
\hline
\end{tabular}

Grupo 7. Processo de Ensino-Aprendizagem

\begin{tabular}{|c|l|l|}
\hline Id. & Métrica & Descrição \\
\hline G7.17 & Metodologia de ensino- & Inclusão de proposta de metodologia de uso do simulador para apoio \\
\hline
\end{tabular}




\begin{tabular}{|l|l|l|} 
& aprendizagem & ao aprendizado em AOC \\
\hline G7.18 & $\begin{array}{l}\text { Materiais didáticos } \\
\text { complementares }\end{array}$ & $\begin{array}{l}\text { Avaliabilidade de outros tipos de mídias de apoio ao aprendizado } \\
\text { em AOC, como tutoriais, slides de aulas, videoaulas, exemplos de } \\
\text { programas em linguagem de baixo nível, etc. }\end{array}$ \\
\hline G7.19 & Canal de apoio & $\begin{array}{l}\text { Disponibilidade de canal de comunicação para discussões entre } \\
\text { desenvolvedores e usuários (docentes e estudantes). }\end{array}$ \\
\hline
\end{tabular}

\section{Discussão e Análise dos Resultados}

A Tabela 1, a seguir, sumariza o resultado do estudo comparativo entre simuladores. $\mathrm{Na}$ Tabela 1, os simuladores estão dispostos, na coluna mais à esquerda, em grupos relacionados aos seus objetivos principais: S1) educacional, S2) suporte a sistema operacional, S3) descrito em HDL e S4) suporte à computação intensiva. Nas demais colunas, estão dispostos os grupos de métricas (G1 ao G7), com as respectivas métricas.

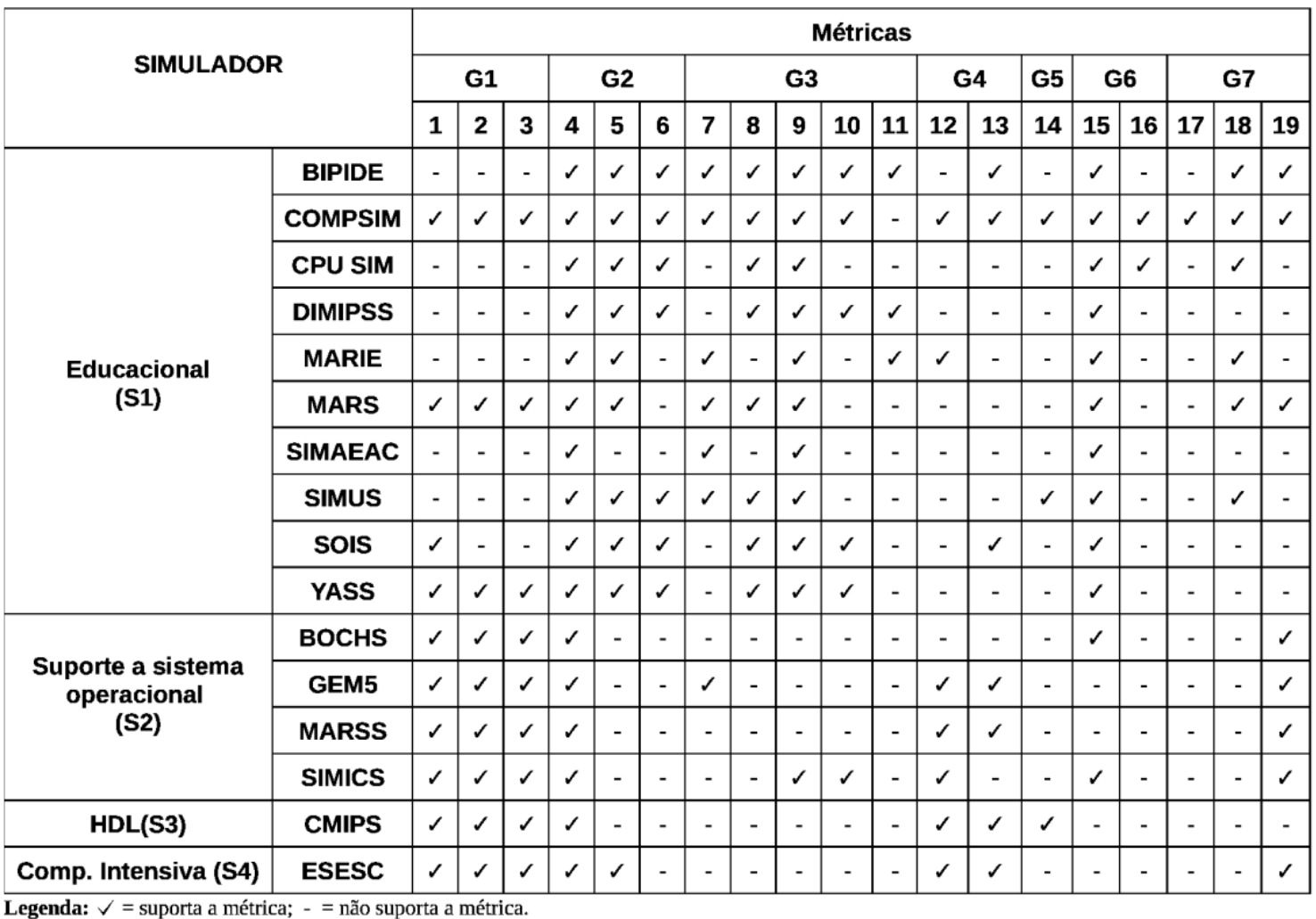

Tabela 1. Comparativo entre simuladores de sistemas completos.

Analisando a Tabela 1, pode-se constatar que os macrotópicos da disciplina de AOC, que são o estudo dos componentes do computador, programação em nível de máquina e análise de desempenho, apontados pela SBC e, neste estudo, cobertos pelos grupos de métricas G1, G2 e G4, respectivamente, são parcialmente tratados pelos simuladores.

Os simuladores do grupo S1, não suportam, de uma forma geral, as métricas do grupo G1. Eles buscam estabelecer um compromisso entre o detalhamento dos componentes simuláveis e os aspectos de simulação para favorecer o processo de aprendizado pelo acompanhamento da execução do sistema computacional, cujas métricas estão presentes e parcialmente cobertas no grupo G3 (Simulação). Por outro 
lado, os simuladores dos demais grupos (S2 ao S4) suportam todas as métricas do G1, objetivando fidelizar as características do hardware simulado para favorecer seus objetivos individuais (suportar execução de sistemas operacionais, execução em hardware e simulação de aplicações de alto de desempenho e precisão, respectivamente). Com isso, nos grupos $\mathrm{S} 2$ ao $\mathrm{S} 4$, prejudica-se o suporte às métricas do grupo G3.

Analisando o grupo de métricas G2, percebe-se que todos os simuladores avaliados suportaram a métrica 4 (Programação em Assembly), o que mostra uma preocupação no aprendizado dos tópicos de arquitetura de computadores e suporte à execução de aplicações reais em nível de máquina. As métricas 5 e 6, do grupo G2, estão diretamente associadas ao suporte ao aprendizado em programação em nível de máquina, sendo estas quase que completamente suportadas pelos simuladores em S1 e não suportados pelos demais grupos.

O suporte à análise de desempenho, um dos três macrogrupos de tópicos elencados para a disciplina de AOC pela SBC, tratada pelas métricas do grupo G4, é favorecido pelos simuladores em S2 ao S4. Percebe-se, nesse sentido, uma carência de recursos para aprendizado em otimização de desempenho computacional nos simuladores em S1.

Já o suporte ao desenvolvimento de habilidades práticas, através da integração do simulador com dispositivos físicos de hardware, coberto pela métrica 14, em G5, está sendo tratado por apenas 3 simuladores, sendo dois deles do grupo S1 (COMPSIM e SIMUS) e um do S3 (CMIPS). Observa-se que, para a integração, foram consideradas as diferentes abordagens, sendo elas: execução do simulador em sistema computacional embarcado (SIMUS), uso de plataforma aberta de prototipação como periférico (COMPSIM) e execução em FPGA (CMIPS). Esses resultados remetem à necessidade de mais esforços para suportar essa área pelos simuladores, visto que está diretamente relacionada à aplicação dos conceitos da disciplina de $\mathrm{AOC}$ e desenvolvimento das respectivas habilidades práticas.

Em termos de acessibilidade e usabilidade (métricas do grupo G6), todos os simuladores em S1 e metade dos em S2 incluem interfaces gráficas e, de uma forma geral, não são personalizáveis. Nesse sentido, percebe-se que, para todos os simuladores, são necessários mais estudos na área de Interação Homem-Máquina (IHM), pois ressalta-se que o uso de interfaces gráficas é um recurso importante que pode simplificar e maximizar as habilidades de uso do simulador, bem como trazer um feedback mais rápido e natural sobre as ações realizadas. Da mesma forma, a personalização da interface permite aumentar os níveis de compreensão das operações do simulador além de trazer experiências mais agradáveis.

Analisando o último grupo de métricas (G7), o qual está relacionado diretamente ao processo de ensino-aprendizagem, percebe-se uma grande deficiência, pois os simuladores, em quase sua totalidade, não oferecem uma metodologia de apoio ao uso de simulador nas aulas da disciplina de AOC. Os materiais didáticos complementares, na grande maioria, tratam de aspectos de simulação e/ou estão relacionados diretamente ao simulador, e não em aprendizagem em AOC, bem como muitos dos simuladores não oferecem canais de comunicação, em que poderiam ser estabelecidos diálogos com desenvolvedores e entre usuários, para maximizar o aprendizado, compartilhar experiências do uso do simulador, bem como sugerir e discutir modificações e inclusão de novos recursos pedagógicos.

A Figura 1 traz gráficos de rede que tratam cada grupo de simuladores e o percentual de atendimento a cada métrica dos grupos G1 ao G7. 


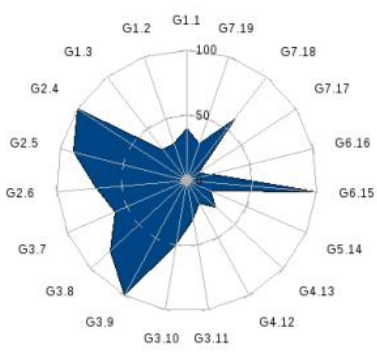

(a) Distribuição de S1.

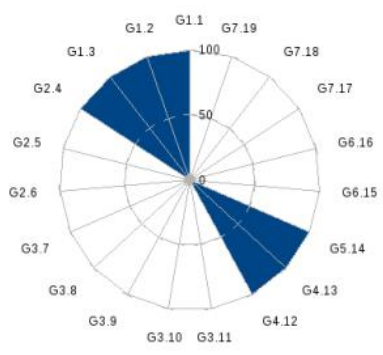

(c) Distribuição de S3.

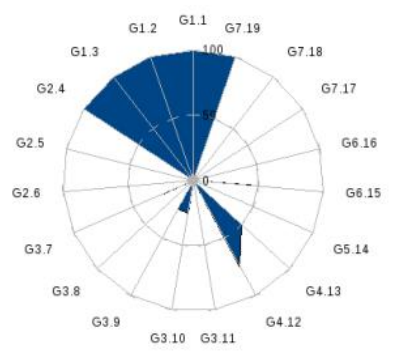

(b) Distribuição de S2.

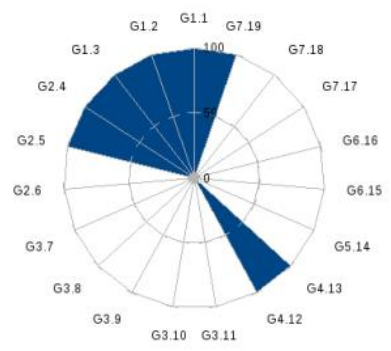

(d) Distribuição de S4.

Figura 1. Gráficos de rede: distribuição de métricas por grupos de simuladores.

No gráfico da Figura 1(a), observa-se que a maior parte dos simuladores do grupo $\mathrm{S} 1$ buscaram atender às métricas contidas nos grupos $\mathrm{G} 2$ (programação em baixo nível) e G3 (simulação), e, uma pequena parte, às métricas dos grupos G1 (componentes do computador), G6 (acessibilidade e usabilidade) e G7 (processo de ensinoaprendizagem). Ao analisar os gráficos das Figuras 1(b), 1(c) e 1(d), percebe-se que os simuladores dos grupos $\mathrm{S} 2, \mathrm{~S} 3$ e $\mathrm{S} 4$ buscaram atender às métricas dos grupos $\mathrm{G} 1$ (componentes do computador), às métricas 12 e 13 do G4 (análise de desempenho), e à métrica 4 (programação em Assembly) do grupo G2. Dessa análise, percebe-se que os simuladores do grupo $\mathrm{S} 1$, ao incluírem um maior número de recursos didáticos, tornamse melhores candidatos para apoio às disciplinas de AOC. Entretanto, percebe-se que, de um forma geral, esse grupo apresenta várias deficiências - tais como, a ausência de maior granularidade dos componentes simulados, suporte à análise de desempenho e integração com hardware físico - sendo assim necessário realizar uma análise individual dos simuladores.

A Figura 2(a) apresenta um gráfico de barras, onde pode-se visualizar a distribuição de porcentagens de métricas atendidas pelos simuladores.

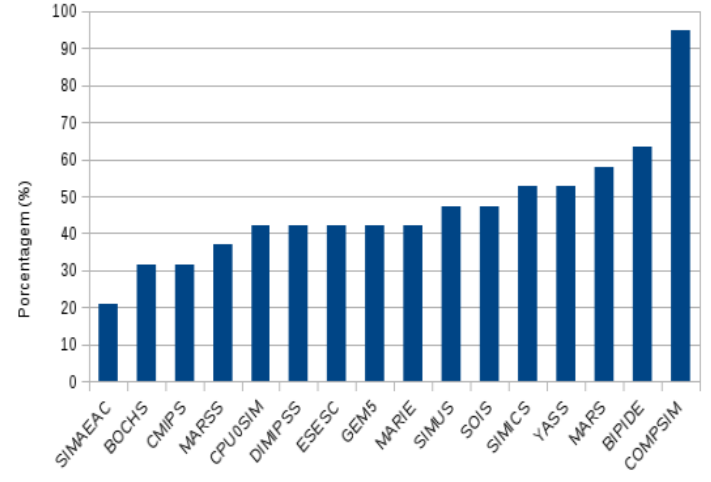

(a) Distribuição de métricas atendidas pelos simuladores

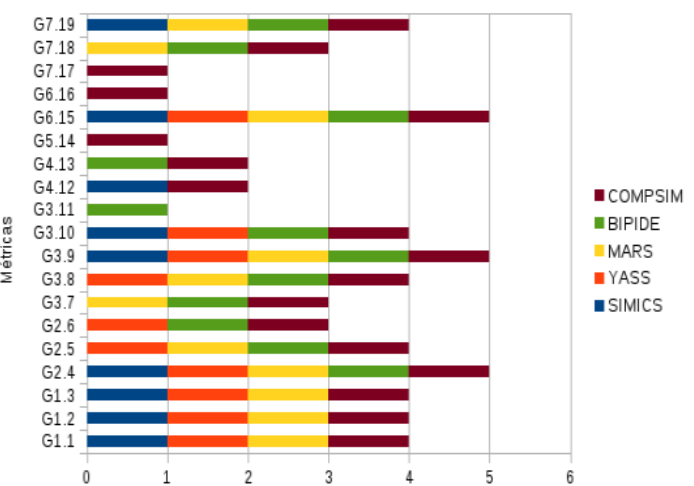

(b) Métricas atendidas pelos simuladores melhores avaliados. 


\section{Figura 2. Gráficos de barras (a) e barras empilhadas (b): simuladores versus métricas atendidas.}

Na Figura 2(a), o gráfico dispõe os simuladores analisados em ordem crescente de acordo com a porcentagem de atendimento às métricas. No gráfico, dentre os simuladores, apenas 5 deles atenderam a mais de $50 \%$ das métricas propostas, onde um deles pertence ao grupo S2 - SIMICS $(52,6 \%)$ - e quatro ao grupo S1, YASS (52,6\%), MARS (57,8\%), BIPIDE (63,1\%) e COMPSIM (94,7\%). Já a Figura 2(b) apresenta um gráfico de barras empilhadas, onde pode-se verificar quais métricas foram atendidas por cada um desses cinco simuladores.

Na Figura 2(b), as métricas 4 (programação em Assembly), 9 (visualização dos estados dos componentes) e 15 (interface gráfica) foram atendidas pelos 5 simuladores e, consequentemente, podem ser caracterizadas como fundamentais a um bom simulador para apoio à disciplina de AOC. Por outro lado, analisando as métricas que tiveram menor cobertura, percebe-se a existência de lacunas no apoio ao processo de ensino-aprendizagem e, consequentemente, necessitam de emprego de maiores esforços, visto que, as métricas 11 (animação do caminho de dados) e 16 (personalização da interface) estão ligadas à acessibilidade, bem como as métricas 14 (integração com hardware físico) e 17 (metodologia de ensino-aprendizagem) estão diretamente relacionadas à abordagem pedagógica em AOC.

\section{Conclusões}

Arquitetura e organização de computadores é uma disciplina com conteúdos extensos e complexos, bem como necessita de laboratórios especializados para suporte ao desenvolvimento das habilidades práticas. Visando dirimir a dificuldade em compor tais laboratórios, que são caros e necessitam de apoio técnico especializado, na literatura, tem-se optado pelo uso dos simuladores computacionais. Este trabalho realizou um estudo comparativo entre diferentes tipos de simuladores de sistemas completos, que são aqueles que incluem todos os componentes do computador, sob grupos de métricas, elaboradas a partir de estudos comparativos da literatura, nos tópicos base da disciplina, apontados pela SBC, e em aspectos de suporte educacional.

Os resultados mostraram que as métricas propostas neste estudo estão parcialmente tratadas pelos grupos de simuladores estudados. Os simuladores educacionais apresentaram grande potencial em suporte à programação em baixo nível e simulação do computador, enquanto que os demais apresentaram granularidade nos componentes do computador e suporte à análise de desempenho. Dentre os simuladores que trataram a maior parte das métricas, destacaram-se, como métricas predominantes, o suporte à programação em baixo nível, visualização dos estados dos componentes e interface gráfica, enquanto que observou-se a necessidade de mais estudos para suportar aspectos de acessibilidade dos simuladores e metodológicos da disciplina.

\section{Referências}

Akram, A. and Sawalha, L. (2016) "x86 computer architecture simulators: A comparative study". In IEEE 34th International Conference on Computer Design (ICCD).

Ardestani, E. K., Renau, J. (2013) "ESESC: A fast multicore simulator using time-based sampling". In 2013 IEEE 19th International Symposium on High Performance Computer Architecture (HPCA). p. 448-459.

Black, M. (2016) "Export to arduino: a tool to teach processor design on real hardware". In Journal of Computing Sciences in Colleges, 31(6), pp.21-26. 
Bochs. (2017) The Bochs IA-32 Emulator Project. http://bochs.sourceforge.net.

Butko, A., Garibotti, R., Ost, L. and Sassatelli, G. (2012) “Accuracy evaluation of gem5 simulator system". In 7th International Workshop on Reconfigurable and Communication-Centric Systems-on-Chip (ReCoSoC). pp. 1-7.

Cordeiro, E. S., Stefani, I. G., Soares, T. C. and Martins, C. A. (2003) "DCMSim: Didactic cache memory simulator". In 33rd Annual Frontiers in Education. Vol. 2, pp. F1C 14-19.

Cruz, E. H. M., Foleiss, J. H., Assunção, G. P. and Gonçalves, R. A. L. (2008) "Ferramenta de Simulação de Processador para Ensino de Graduação e Pesquisa Científica". In Anais SULCOMP, v. 4.

Donzellini, G. and Ponta, D. (2007) "A simulation environment for e-learning in digital design". In IEEE Transactions on Industrial Electronics, 54(6), pp. 3078-3085.

Esmeraldo, G. and LISBOA, E. B. (2017) "Uma Ferramenta para Exploração do Ensino de Organização e Arquitetura de Computadores". In International Journal of Computer Architecture Education, v. 6. p. 68-75.

Felix, A., Pousa, C., and Carvalho, M. (2006) "DIMIPSS: Um simulador didático e interativo do MIPS”. In Workshop sobre Educação em Arquitetura de Computadores. p. 49-52.

Ghafarian, R. (2016) "Microelectronics packaging technology roadmaps, assembly reliability, and prognostics". Facta universitatis-series: Electronics and Energetics, 29(4), pp.543-611.

Hexsel, R. A. and Carmo, R. (2013) “cMIPS - uma Ferramenta Pedagógica para o Estudo de Arquitetura". In: International Journal of Computer Architecture Education. v.2, n.1. p. 29 -32.

Kleitz, W. (2006) Digital Electronics with VHDL, Quartus II Version. Pearson Prentice Hall.

Kurniawan, W. and Ichsan, M. H. H. (2017) "Teaching and learning support for computer architecture and organization courses design on computer engineering and computer science for undergraduate: A review". In 4th International Conference on Electrical Engineering, Computer Science and Informatics (EECSI). pp. 1-6.

Magnusson, P. S., Christensson, M., Eskilson, J., Forsgren, D., Hallberg, G., Hogberg, J., and Werner, B. (2002) "Simics: A full system simulation platform". In Computer, $35(2)$, p. $50-58$.

Mustafa, B. (2013) "YASS: A System Simulator for Operating System and Computer Architecture Teaching and Learning". In European Journal of Science and Mathematics Education, v. 1, n. 1, p. 34-42, 2013.

Nikolic, B., Radivojevic, Z., Djordjevic, J. and Milutinovic, V. A. (2009) "Survey and Evaluation of Simulators Suitable for Teaching Courses in Computer Architecture and Organization". IEEE Transactions on Education, Vol. 52, No. 4.

Null, L. and Lobur, J. (2003). "MarieSim: The MARIE computer simulator". In Journal on Educational Resources in Computing (JERIC), 3(2), 1.

Patel, A., Afram, F., Chen, S. and Ghose, K. (2011) "MARSS: a full system simulator for multicore x86 CPUs". In 2011 48th ACM/EDAC/IEEE Design Automation Conference (DAC). pp. 1050-1055.

Penna, P. H. M. M. and Freitas, H. C. (2013) "Análise e Avaliação de Simuladores de 
Sistemas Completos para o Ensino de Arquitetura de Computadores". International Journal of Computer Architecture Education (IJCAE), v. 2, no. 1. pp 13-16.

Rocha, M. da G. B., Nicoletti, M. do C., Fabbri, S. C. P. F., Barros, E. N. da S. and Frery, A. C. (2005) "Currículo de Referência da SBC para Cursos de Graduação em Bacharelado em Ciência da Computação e Engenharia de Computação". Relatório Técnico, Sociedade Brasileira de Computação (SBC).

Silva, G. P. and Borges, J. A. dos S. (2017) "O Simulador SimuS na Plataforma Raspberry Pi”. In Computer Architecture Education (IJCAE) v. 6, n. 1. p. 36-45.

Skrien, D. (2001) "CPU Sim 3.1: A tool for simulating computer architectures for computer organization classes". In Journal on Educational Resources in Computing (JERIC), 1(4), 46-59.

Verona, A. B., Martini, J. A. and Gonçalves, T. L. (2009) "SIMAEAC: Um Simulador Acadêmico para Ensino de Arquitetura de Computadores". In Varia Scientia, v. 9, n. 16, p. 139-148.

Vieira, P. V., Raabe, A. L. A. and Zeferino, C. A. (2009) "Bipide: Ambiente de desenvolvimento integrado para utilização dos processadores bip no ensino de programação". XX SBIE.

Vollmar, K. and Sanderson, P. (2006) "MARS: an education-oriented MIPS assembly language simulator”. In SIGCSE, Vol. 6. pp. 239-243.

Wolffe, G. S., Yurcik, W., Osborne, H. and Holliday, M. A. (2002) "Teaching computer organization/architecture with limited resources using simulators". In Proceedings of the 33rd SIGCSE technical symposium on Computer science education, ACM SIGCSE Bulletin. Vol. 34, No. 1. 